INPLASY

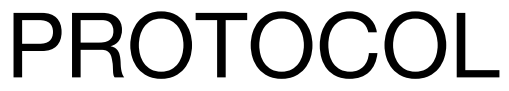

To cite: Chai et al. Influence of dipeptidyl peptidase-4 inhibitors on glycemic variability in patients with type 2 diabetes: a meta-analysis of randomized controlled trials. Inplasy protocol 2021120113. doi:

10.37766/inplasy2021.12.0113

Received: 24 December 2021

Published: 24 December 2021

Corresponding author: Miao Yu

yumiaoxh@163.com

Author Affiliation:

Department of Endocrinology, Key Laboratory of

Endocrinology, National Health Commission, Peking Union Medical College Hospital, Peking Union Medical College and Chinese Academy of Medical Sciences, Beijing, 100010, China.

Support: Without financial support.

Review Stage at time of this submission: Data analysis.

\section{Influence of dipeptidyl peptidase-4 inhibitors on glycemic variability in patients with type 2 diabetes: a meta- analysis of randomized controlled trials}

Chai, S1; Zhang, R2; Zhang, Y3; Carr, RD4; Zheng, Y5; Rajpathak, S6; Yu, M?.

Review question / Objective: In this study, we aimed to perform an updated meta-analysis to summarize the current knowledge regarding the influence of DPP4 inhibitors on glycemic variably as compared with other OADs compared to the previous meta-analysis. Moreover, with more available RCTs, this meta-analysis is expected to provide the relative efficacy of DPP4 inhibitors on MAGE as compared with individual categories of OADs, including the sodium-glucose cotransporter 2 (SGLT2) inhibitors.

Condition being studied: Some small-scale randomized controlled trials (RCTs) have been performed to compare the influences of DPP4 inhibitors and other OADs on MAGE in T2DM patients, the results of these RCTs were not consistent and a consensus on the efficacy of DPP4 inhibitors on glycemic variability compared to other OADs has not been fully determined. A previous meta-analysis including only seven RCTs before 2018 showed that DPP4 inhibitors may be more effective than other OADs in reducing MAGE. However, some recently published eligible studies have not been included in a meta-analysis.

INPLASY registration number: This protocol was registered with the International Platform of Registered Systematic Review and Meta-Analysis Protocols (INPLASY) on 24 December 2021 and was last updated on 24 December 2021 (registration number INPLASY2021120113).

\section{INTRODUCTION}

Review question / Objective: In this study, we aimed to perform an updated meta- analysis to summarize the current knowledge regarding the influence of DPP4 inhibitors on glycemic variably as compared with other OADs compared to 
the previous meta-analysis. Moreover, with more available RCTs, this meta-analysis is expected to provide the relative efficacy of DPP4 inhibitors on MAGE as compared with individual categories of OADs, including the sodium-glucose cotransporter 2 (SGLT2) inhibitors.

Condition being studied: Some small-scale randomized controlled trials (RCTs) have been performed to compare the influences of DPP4 inhibitors and other OADs on MAGE in T2DM patients, the results of these RCTs were not consistent and a consensus on the efficacy of DPP4 inhibitors on glycemic variability compared to other OADs has not been fully determined. A previous meta-analysis including only seven RCTs before 2018 showed that DPP4 inhibitors may be more effective than other OADs in reducing MAGE. However, some recently published eligible studies have not been included in a meta-analysis.

\section{METHODS}

Participant or population: (1T2DM patients without concurrent insulin treatment(2) Either drug naive, or on the same background therapy.

Intervention: (1)Oral DPP-4 inhibitors with approved doses(2)Including sitagliptin, vildagliptin, linagliptin, saxagliptin, alogliptin, dutogliptin, aemigliptin, anagliptin, teneligliptin, trelagliptin, omarigliptin, gemigliptin, or evogliptin.

\section{Comparator: Other OADs.}

Study designs to be included: RCTs without restriction on blindness.

Eligibility criteria: Studies that fulfilled the following criteria were included: (1) fulllength articles published in English; (2) designed as parallel-group or crossover RCTs; (3) included adult patients with T2DM who were not treated with insulin; (4) patients were allocated to a treatment group with DPP4 inhibitors or a control group with other OADs; and (5) reported the between-group difference of changes in MAGE using CGM or FGM from the baseline for patients in each arm of the study. Studies with drug-naïve patients or T2DM patients on background OAD therapy were included. Studies including T2DM patients with concurrent insulin therapy were excluded because the influence of insulin on blood glucose fluctuations is related to various factors such as dosage and categories, which may conceal the effect of combined OADs on blood glucose fluctuations. We did not consider studies including patients treated with single-dose/ single-day DPP4 inhibitors because we did not plan to evaluate the acute effect of DPP4 inhibitors on glycemic variability. In addition, non-randomized studies, studies with non-T2DM patients, and those without MAGE measurement using CGM or FGM were excluded.

Information sources: Medline (PubMed), Embase (Ovid), and CENTER (Cochrane Library) databases were searched for relevant studies with the following keywords: (1) "DPP4" OR "DPP-4" OR "dipeptidyl peptidase-4 inhibitors" OR "sitagliptin" OR "vildagliptin" OR "linagliptin" OR "saxagliptin" OR "alogliptin" OR "dutogliptin" OR "aemigliptin" OR "anagliptin" OR "teneligliptin" OR "trelagliptin" OR "omarigliptin" OR "gemigliptin" OR "evogliptin"; (2) "continuous glucose monitoring" OR "glycemic variability" OR "glyceamic variability" OR "glucose variability" OR "glucose fluctuation" OR "glycemic fluctuation" OR "mean amplitude of glycemic excursion" OR "MAGE" OR "standard deviation" OR "SD" OR "SDBG" OR "largest amplitude of glycemic excursion" OR "LAGE" OR "Coefficient of variation" OR "CV"; and (3) "random" OR "randomized" OR "randomised" OR "randomly". Only clinical studies were considered. The references of related reviews and original articles were also searched as a complement. The final database search was conducted on July 23, 2021.

Main outcome(s): The primary outcome is to quantitatively compare the influences of 
DPP4 inhibitors versus other OADs on glycemic variably as evaluated by MAGE in T2DM patients without concurrent insulin therapy. The secondary outcome is to explore the relative efficacy of DPP4 inhibitors on MAGE as compared with insulin secretagogues (SUs and glinides), non-secretagogues, and individual categories of OADs.

Quality assessment / Risk of bias analysis: For the studies included the validity of individual trials will be assessed using the Risk of Bias instrument, endorsed by the Cochrane Collaboration, as specifie. This instrument is used to evaluate 6 key domains: sequence generation; allocation concealment; blinding of participants, personnel and outcome assessors; incomplete outcome data; selective outcome reporting; and other sources of bias. In addition to the 6 key components, an assessment of the study designs of the included trials will also be conducted to determine whether additional quality components should be included in the bias risk assessment. The risk of bias instrument can be used to assign summary assessments of within study bias; low risk of bias, unclear risk of bias, or high-risk of bias. The potential for publication bias will be examined by using a funnel plot and statistical tests for asymmetry. A funnel plot displays trial treatment effect estimates ( $x$ axis) against some measure of their precision such as standard error ( $y$ axis). When no publication bias is present the plot should show a funnel-like shape, with estimates spanning down from the larger trials symmetrically in both directions with increasing variability. Asymmetry in a funnel plotis potentially indicative of publication biases, but other sources of heterogeneity may also induce asymmetry in a funnel plot. If there is asymmetry and studies are perceived to be missing in those regions of non-statistical significance, there is greater likelihood that the asymmetry is due to publication bias. For each funnel plot, we will test for asymmetry with the rank correlation test and Egger regression, and a $P$ value $<0.10$ will indicate statistical evidence of asymmetry. In addition to statistically assessing publication bias, it is important to note that our literature search included abstracts as well as publications in order to more fully capture the available literature and minimize this type of bias. We will employ the GRADE system for rating the overall quality of evidence. For each outcome, randomized trials begin as highquality evidence, but may be rated down by one or more of five categories of limitations: (1) risk of bias, (2) consistency, (3) directness, (4) imprecision, and (5) publication bias. The quality of evidence for each main outcome can be determined after considering each of these elements, and categorized as either high, moderate, low, or very low.

Strategy of data synthesis: 1 Statistical Analysis for Pairwise Meta-Analysis Between-group difference of changes of MAGE using CGM or FGM from baseline was used as the outcome of interest. The pooled effect was presented as mean difference (MD) with $95 \%$ confidence intervals $(\mathrm{Cl})$. To calculate the overall difference between the two periods, each study was weighted by the reciprocal of the variance for MAGE change. In a conservative approach, the random-effect estimates of mean differences, which allow for variation of true effects across studies, were taken as 'main results'. Because variances for MAGE change between baseline and final measurements were not directly reported, they were calculated by assuming a correlation coefficient of 0.5. 2 Assessment of statistical heterogeneity Heterogeneity across trial within comparator subgroups will be quantitatively assessed using the conventional measure of heterogeneity, 12, as well as the Cochrane's $Q$ test for homogeneity. However, as only few studies are expected to be present within each subgroup, these quantitative tests hold little value. Any differences between studies, subjectively assessed to be potentially important will therefore be examined in further detail by comparing important study design and population characteristics across RCTs. Where more than one trial is available for a specific comparison, pair wise meta-analysis will be 
employed to produce pooled results, i.e., pooled relative risks with $95 \%$ confidence intervals (Cls). As a primary analysis the conventional DerSimonian-Laird randomeffects models will be employed, and as a sensitivity analysis a fixed-effect model will be employed. The meta-analysis will be conducted using RevMan endorsed by Cochrane Collaboration.

Subgroup analysis: Subgroup analyses were will be performed to identify the possible source of heterogeneity; univariate meta-regression will be performed if at least ten datasets were included - Median values of continuous variables were will be used as cut-offs Subgroup analysis will primarily be performed to evaluate the influence of difference in control OADs (Insulin secretagogues or Non-insulin secretagogues; different classes of OADs) on the outcome. Besides, additional subgroup analyses will include variables of other characteristics of the study, such as study design, sample size, proportions of males, mean baseline HbA1c, whether the T2DM patients were drug naïve or not, CGM/FGM analysis for MAGE, and followup durations.

Sensitivity analysis: Sensitivity analysis by omitting one study at a time will be performed to evaluate the robustness of the findings. Moreover, sensitivity analyses will also be performed in studies of doubleblind RCTs, in studies including drug naïve patients only, in studies with insulin secretagogues as controls, and in studies with non-secretagogues as controls. Besides, multiple sensitivity analyses will be performed according to criteria if needed, such as sensitivity analysis according to study design, sample size, age stratification, proportions of males, mean baseline $\mathrm{HbA1c}$, duration of diabetes, and whether the T2DM patients were drug naïve or not.

\section{Country(ies) involved: China.}

Keywords: dipeptidyl peptidase-4 inhibitor; glycemic variability; mean amplitude of glycemic excursion; type 2 diabetes mellitus; meta-analysis.

\section{Contributions of each author:}

Author 1 - Shangyu Chai - conceived and designed research; collected data and conducted research; performed or supervised analyses; interpreted data; wrote the initial paper. provided substantive suggestions for revision, reviewed and approved final version of the paper.

Author 2 - Ruya Zhang - conceived and designed research; collected data and conducted research; interpreted data; provided substantive suggestions for revision, reviewed and approved final version.

Author 3 - Ye Zhang - conceived and designed research; provided substantive suggestions for revision, reviewed and approved final version.

Author 4 - Richard David Carr - conceived and designed research; provided substantive suggestions for revision, reviewed and approved final version.

Author 5 - Yiman Zheng - interpreted data; provided substantive suggestions for revision, reviewed and approved final version.

Author 6 - Swapnil Rajpathak - interpreted data; provided substantive suggestions for revision, reviewed and approved final version.

Author 7 - Miao Yu - conceived and designed research; performed or supervised analyses; interpreted data; SYC wrote the initial paper. provided substantive suggestions for revision, reviewed and approved final version.

Conflicts of interest: Shangyu Chai, Ruya Zhang, Ye Zhang and Yiman Zheng are employees of MSD China; Rajpathak Swapnil is employee of Merck Sharp \& Dohme Corp., a subsidiary of Merck \& Co., Inc., Kenilworth, NJ, USA; Other authors have none to declare. 